\title{
Rich Club Organization and Cognitive Performance in Healthy Older Participants
}

\author{
Hugo C. Baggio ${ }^{1,2}$, Barbara Segura ${ }^{1}$, Carme Junque ${ }^{1,2}$, Marcel A. de Reus ${ }^{3}$, \\ Roser Sala-Llonch ${ }^{1}$, and Martijn P. Van den Heuvel ${ }^{3}$
}

\begin{abstract}
The human brain is a complex network that has been noted to contain a group of densely interconnected hub regions. With a putative "rich club" of hubs hypothesized to play a central role in global integrative brain functioning, we assessed whether hub and rich club organizations are associated with cognitive performance in healthy participants and whether the rich club might be differentially involved in cognitive functions with a heavier dependence on global integration. A group of 30 relatively older participants (range $=39-79$ years of age) underwent extensive neuropsychological testing, combined with diffusion-weighted magnetic resonance imaging to reconstruct
\end{abstract}

\section{INTRODUCTION}

Cognitive brain functions depend on the integrated activity between distinct but anatomically connected functional subunits, together forming a highly complex brain network (Bressler \& Menon, 2010; van den Heuvel \& Hulshoff Pol, 2010; Bullmore \& Sporns, 2009). Recent advances in the field of connectomics have shed light on the organizational properties of structural and functional brain networks, thought to be part of the neural bases of cognitive processing (McIntosh, 2000).

Graph theory forms a powerful framework for the description of quantifiable network parameters that can be used as neurobiologically meaningful measures (Bullmore \& Sporns, 2009). Under this approach, macroscale structural brain networks have been described as graphs in which anatomical brain regions are described as nodes, linked by edges describing structural connections of white matter (WM) tracts. Given this mathematical framework, a node's degree is defined as the number of edges linked to it, indicating its accessibility within the network. Degree in neural networks has been noted to have a heavy-tailed distribution, denoting the presence of a set of highly connected nodes (Hagmann et al., 2008). These putative "neural hubs" are hypothesized to play a central

${ }^{1}$ University of Barcelona, ${ }^{2}$ CIBERNED, Barcelona, ${ }^{3}$ University Medical Center Utrecht individual structural brain networks. Rich club connectivity was found to be associated with general cognitive performance. More specifically, assessing the relationship between the rich club and performance in two specific cognitive domains, we found rich club connectivity to be differentially associated with attention/executive functions-known to rely on the integration of distributed brain areas-rather than with visuospatial/visuoperceptual functions, which have a more constrained neuroanatomical substrate. Our findings thus provide first empirical evidence of a relevant role played by the rich club in cognitive processes. role in overall network communication and intermodular information transfer (van den Heuvel \& Sporns, 2013b; Sporns, Honey, \& Kötter, 2007). Moreover, this set of hubs appears to be more highly connected to other highdegree nodes than predicted by chance, forming a group of highly interconnected regions (i.e., the "rich club") that may act as a backbone for global network integration (De Reus, Saenger, Kahn, \& van den Heuvel, 2014; Mišić, Sporns, \& McIntosh, 2014; Towlson, Vértes, Ahnert, Schafer, \& Bullmore, 2013; Van den Heuvel, Kahn, Goñi, \& Sporns, 2012). Functionally, the anatomical rich club is also hypothesized to act as a gatekeeper, modulating the dynamical interactions between lower-degree regions and the emergence of distinct functional network configurations (Senden, Deco, de Reus, Goebel, \& van den Heuvel, 2014; Crossley et al., 2013).

Here, we investigated the role of the rich club in cognitive performance in a sample of healthy older participants. We hypothesize the rich club connectivity to be associated with overall cognitive functioning, in particular with performance in specific cognitive functions related to more distributed anatomical correlates. To test this hypothesis, we assessed the relationship between rich club organization and global cognitive performance and compared structural connectivity correlates of two cognitive domains-attention/executive (A/E) and visuospatial/ visuoperceptual (VS/VP) functions - with putatively higher and lower reliance on the integration of distributed brain areas, respectively. 


\section{METHODS}

Thirty adult participants ( 14 women; age: mean $=66.2$ years, $S D=10.4$ years, range $=39-79$ years; right-handed) without cognitive complaints were recruited from individuals who volunteered to participate in scientific studies at the Institut de l'Envelliment, Universitat Autònoma de Barcelona. Exclusion criteria were history of psychiatric, neurological or systemic diseases, or pathological MRI findings other than mild WM hyperintensities in the FLAIR sequence. The study was approved by the ethics committee of the University of Barcelona; all participants provided written informed consent to participate.

\section{Neuropsychological Assessment}

Participants were administered a neuropsychological battery with tests addressing main cognitive functions such as attention, executive functions, VS/VP function, memory, and language (see Table 1 ; for a detailed description of the tests used, see Lezak, 2004). $z$ scores for each test and participant were calculated based on the group's means and standard deviations. Composite $z$ scores for general cognition (general cognition scores) were calculated as the mean $z$ scores of all tests used. Domain-specific composite $z$ scores (A/E scores, VS/VP scores) were calculated as the mean $z$ scores of all tests within the respective domains, as shown in Table 1.

\section{MRI Acquisition and Preprocessing}

Images were obtained with a 3-T MRI scanner (MAGNETOM Trio, Siemens, Berlin, Germany). The scanning protocol

Table 1. Neuropsychological Assessment Performance

\begin{tabular}{lc}
\hline Backward minus forward digit spans $^{\mathrm{a}}$ & $-1.27(1.05)$ \\
Trail-Making Test Part A minus Part B (sec) $^{\mathrm{a}}$ & $-52.76(24.33)$ \\
Phonemic fluency $^{\mathrm{a}}$ & $17.00(4.39)$ \\
Stroop Color-Word Test interference $^{\mathrm{a}}$ & $-0.93(7.88)$ \\
Benton's Visual Form Discrimination $^{\mathrm{b}}$ & $30.77(1.38)$ \\
Benton's Judgment of Line Orientation $^{\mathrm{b}}$ & $25.17(3.16)$ \\
RAVLT total learning & $47.27(8.36)$ \\
RAVLT 20-min free recall & $10.50(2.50)$ \\
Boston Naming Test (short version) & $14.07(.98)$ \\
Vocabulary subtest (Wechsler Adult Intelligence & $47.41(8.39)$ \\
$\quad$ Scale, 3rd edition) & \\
\hline
\end{tabular}

Results are in mean $(S D)$. Phonemic fluency $=$ number of words beginning with "p" produced in 60 sec; RAVLT = Rey's Auditory Verbal Learning Test.

${ }^{\mathrm{a}}$ Tests included in the $\mathrm{A} / \mathrm{E}$ domain.

${ }^{\mathrm{b}}$ Tests included in the VS/VP domain. included a high-resolution three-dimensional T1-weighted sequence acquired sagittally (repetition time [TR]/echo time $[\mathrm{TE}] /$ inversion time $=2300 / 2.98 / 900 \mathrm{msec} ; 256 \times$ 256 matrix, with 1-mm isotropic voxels). Two diffusionweighted datasets were obtained using a single-shot EPI sequence $(\mathrm{TR} / \mathrm{TE}=5533 / 88 \mathrm{msec})$, with diffusion-encoding in 30 directions at $b=1000 \mathrm{sec} / \mathrm{mm}^{2}$ and one volume at $b=0 \mathrm{sec} / \mathrm{mm}^{2}$, with 2-mm isotropic voxels. A T2-weighted axial FLAIR sequence $(\mathrm{TR} / \mathrm{TE}=9 / 96 \mathrm{msec})$ was also acquired.

T1-weighted images were used to parcellate the brain cortex into 68 regions using FreeSurfer V5.1.0 (surfer. nmr.mgh.harvard.edu/; Fischl et al., 2004). Diffusionweighted imaging was performed to reconstruct the WM tracts, collectively forming the structural brain network. After eddy-current distortion and motion correction, each voxel's diffusion profile was fitted a tensor using a robust fitting method (Chang, Walker, \& Pierpaoli, 2012). From the fitted tensors, the fractional anisotropy (FA, taken as a measure of microstructural WM organization) was derived for each voxel. WM tracts were then reconstructed using Fiber Assignment by Continuous Tracking (Mori \& van Zijl, 2002), with eight seeds started in each WM voxel and streamlines tracked along the main diffusion direction from voxel to voxel and stopped when reaching a voxel with $\mathrm{FA}<0.1$, exceeding the brain mask, and/or making a turn $>45^{\circ}$.

\section{Network Computation and Parameters}

With each of the 68 cortical regions describing a node, the edge between nodes $i$ and $j$ was defined by the presence of streamlines touching both regions and weighted by the number of such streamlines. Nodes $i$ and $j$ were considered not to be connected if no streamlines were found between them. Individual $68 \times 68$ connectivity matrices were generated, representing all regions and interconnecting edges.

Nodal and global network parameters were analyzed using the Brain Connectivity Toolbox (Rubinov \& Sporns, 2010). Nodal measures included degree (the number of edges linked to a node) and its weighted version, strength (calculated as the sum of the weights of all edges linked to a node). The global measures included the network density, defined by the total number of edges divided by the total number of possible edges, its weighted version (network strength), and the global efficiency, a metric related to network integration. The global efficiency is inversely related to the average of shortest topological paths between all pairs of nodes (Bullmore \& Sporns, 2009). The topological distance between nodes $i$ and $j$ was defined by the inverse of the number of streamlines identified between them.

Rich club organization was assessed by means of the weighted rich club coefficient $\left(\phi^{\mathrm{w}}(k)\right)$, calculated at the individual level across a range of $k$ levels (Opsahl, Colizza, Panzarasa, \& Ramasco, 2008). Briefly, to calculate the $\phi^{\mathrm{w}}(k)$ 
for a given $k$ level, the number of edges between nodes with degree $>k(n)$ are summed (rich-club $\left.b_{\text {strength }}\right)$. Subsequently, all network edges are ranked, and the $n$ strongest ones are summed (top- $\left.n_{\text {strengtb }}\right)$. The $\phi^{\mathrm{w}}(k)$ is given by the quotient $r i c h$-club $b_{\text {strength }} /$ top- $_{\text {strength }}$. Additionally, the $\phi^{\mathrm{w}}(k)$ is normalized to the averaged $\phi^{\mathrm{w}}(k)$ of a 1000 random networks with equal density and degree distribution. Normalized values of $>1$ over a range of $k$ levels define the presence of rich club organization (Opsahl et al., 2008), indicating that nodes with higher degrees tend to be interconnected by stronger edges than would be predicted by chance.

For subsequent analyses, the top 10 (15\%) nodes in group-averaged node degree were considered to be part of the rich club (Collin, Kahn, de Reus, Cahn, \& van den Heuvel, 2014; Van den Heuvel et al., 2013). On the basis of the definition of rich club nodes described above, network edges were divided into three classes: rich club connections (between rich club nodes), feeder connections (between rich club and non-rich club nodes) and local connections (between non-rich club nodes; De Reus et al., 2014).

\section{Statistical Analysis}

The relationship between network parameters and performance in each cognitive domain was assessed through partial correlations, controlling for age and sex. In analyses involving $\mathrm{A} / \mathrm{E}$ and VS/VP scores, mean scores of all tests not included in the respective cognitive domains were also included as covariates of no interest.

A nonparametric, permutation-based one-sample $t$ test was used to test the $k$ levels at which $\phi^{\mathrm{w}}(k)$ was $>1$. Through random sign-flipping (10,000 iterations), a null distribution of $t$ values was generated, against which the actual $t$ statistic was compared. To assess the significance of cognition vs. $\phi^{\mathrm{w}}(k)$ correlations and to compare them between domains, we evaluated the areas under the group level correlation curve (AUC; Cao et al., 2013) across the $k$ levels at which $\phi^{\mathrm{w}}(k)$ was significantly $>1$. Statistical significance was established through permutation testing (10,000 permutations), randomizing the cognitive/demographic variables and generating a sampling distribution of areas under the curve against which the studied variables were compared.

To assess the importance of rich club nodes in the cognitive functions evaluated, differences between rich club and non-rich club nodal parameters were calculated; to establish their statistical significance, we also used permutation testing, randomizing which 10 nodes belonged to the rich club among all brain nodes. Each actual mean difference was then compared to the sampling distribution of mean differences. Edges present (i.e., at least one interregional streamline) in more than $60 \%$ of participants were included in correlation analyses (De Reus \& van den Heuvel, 2013a).
As a second null hypothesis model, we selected a set of occipital, parietal, and temporo-occipital regions known to be predominantly involved in visual cognition (Konen \& Kastner, 2008a; Milner \& Goodale, 1995), henceforth referred to as "visual network." Regions chosen bilaterally as visual network nodes included four unimodal areas (lateral occipital cortex, pericalcarine cortex, lingual gyrus, and fusiform gyrus) and the superior parietal lobule, a multimodal region (also included in the rich club, compatible with its role in visual attention [Gillebert et al., 2013; Mesulam, 1998]).

Correlation levels between cognitive variables and the strength of nodes and edges within the visual network were compared with those outside the network through permutation testing. Subsequently, to further test the hypothesis of a differential relationship between performance in cognitive functions with more distributed neural substrates and rich club connectivity, we compared the correlation values between cognitive variables and the strength of rich club and visual network nodes through permutation testing. In this analysis, the replacement procedure consisted of randomly assigning rich club and visual network nodes and edges to either network.

Statistical significance was set at $p \leq .05$ (two-tailed, 10,000 permutations), corrected for multiple comparisons by controlling the false discovery rate (FDR) to 5\% (Benjamini, 2010).

\section{RESULTS}

\section{Neuropsychological Assessment}

Participants performed within the normal range in all neuropsychological tests used, according to age-adjusted normative data (Table 1). General cognition scores strongly correlated with both $\mathrm{A} / \mathrm{E}$ and $\mathrm{VS} / \mathrm{VP}$ scores $(r=$ $.76, p<.001$ and $r=.56, p=.001$, respectively). A/E and VS/VP scores were not significantly intercorrelated $(p=.264)$.

Age correlated negatively with general cognition $(r=$ $-.40, p=.029)$ and with VS/VP scores $(r=-.36, p=$ $.052)$ but did not correlate significantly with $\mathrm{A} / \mathrm{E}$ scores $(p=.224)$. Years of education correlated significantly with general cognition $(r=.58, p<.002)$, VS/VP $(r=$ $.31, p=.046)$, and $\mathrm{A} / \mathrm{E}$ performance $(r=.37, p=$ $.024)$. No significant differences in cognitive variables were found between genders.

\section{Global Network Topological Measures}

Age correlated negatively with global network strength $(r=$ $-.52, p=.001)$, with global efficiency $(r=-.50, p=.002)$, and with the strength of rich club and local edges $(r=-.35$, $p=.029$ and $r=-.61, p<.001$, respectively, surviving FDR correction). No significant associations were seen between age and network density, either in the whole network or in any edge class. 
A/E scores correlated with global efficiency $(r=.38$, $p=.048$ without controlling for age, $r=.38, p=.045$ controlling for age) at a low level of significance, not surviving correction for multiple comparisons. No significant correlations were found for other global network metrics, with or without controlling for the effects of age.

\section{Rich Club Connectivity Analysis}

\section{Rich Club Organization}

As shown in Figure 1 , normalized $\phi^{\mathrm{w}}(k)$ were $>1$ between $k$ levels of 16 and 26 ( $p<.050$, FDR-corrected). Importantly, being the main focus of our study, both general cognition and $\mathrm{A} / \mathrm{E}$ performance correlated positively with $\phi^{\mathrm{w}}(k)(\mathrm{AUC}=4.01$, FDR-corrected $p=.009$, and AUC $=3.78$ and $p=.013$, respectively, surviving FDR correction; see Figure 1), driven by significant correla- tions at $k$ levels 16-23 ( $p<.050$, FDR-corrected). VS/VP performance was not significantly correlated with $\phi^{\mathrm{w}}(k)$ (AUC $=-.91, p=.580$ ), and AUC for correlations between both general cognition and $\mathrm{A} / \mathrm{E}$ performance were larger than that for VS/VP (see Figure 1).

The correlations between $\phi^{\mathrm{w}}(k)$ and both general cognition and $\mathrm{A} / \mathrm{E}$ scores suggest that stronger connectivity between rich club nodes (relative to the rest of the brain) correlates with better cognitive performance. Age did not correlate significantly with $\phi^{\mathrm{w}}(k)$ at any $k$ levels.

The 10 nodes with highest-ranking degree at the group level, defined as the rich club, and their respective degrees are shown in Figure 1. Absolute strength in the rich club subnetwork correlated significantly with $\mathrm{A} / \mathrm{E}$ performance (Figure 2). Correlation between general cognition scores and strength in the rich club subnetwork did not reach the significance threshold set $(r=.37, p=.049)$. No significant correlation was found between rich club

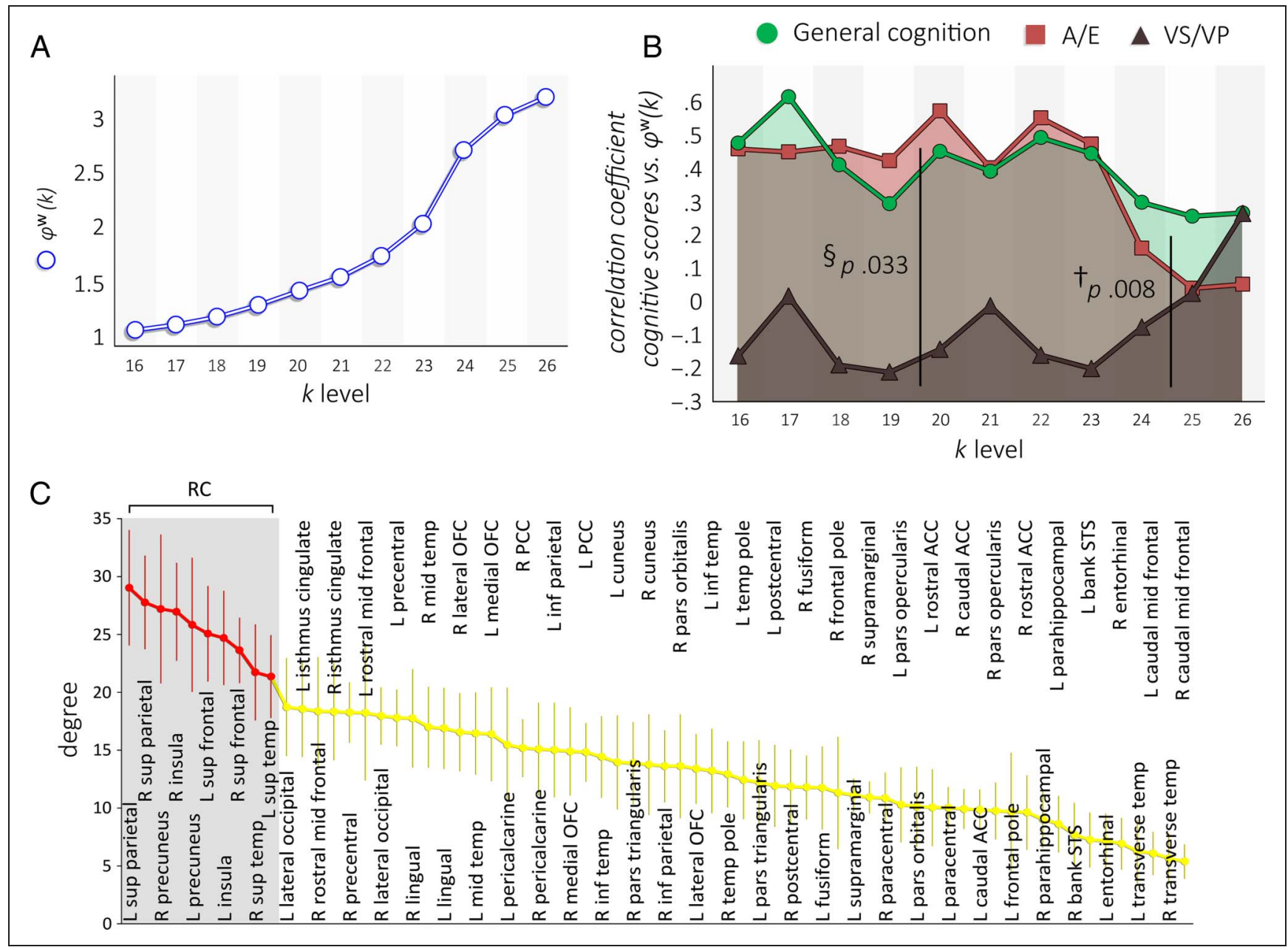

Figure 1. Rich club coefficient $\left(\phi^{\mathrm{w}}(k)\right)$ and rich club (RC) definition. (A) The normalized group-averaged $\phi^{\mathrm{w}}(k)$ curve across the $k$ levels at which it was significantly $>1\left(p<.05\right.$, FDR-corrected). (B) The results of correlation analyses between individual $\phi^{\mathrm{w}}(k)$ and general cognition, A/E and VS/VP performance scores. $p$ values for significant ( $p \leq .05$, FDR-corrected) cross-domain comparisons between areas under the curve of correlation coefficients are shown $(\S=\mathrm{A} / \mathrm{E}$ greater than VS/VP; $\dagger=$ general cognition greater than VS/VP). (C) Group level node degree values are shown in descending order. The 10 top-ranking nodes were defined as the rich club. OFC = orbitofrontal cortex; ACC = anterior cingulate cortex; PCC $=$ posterior cingulate cortex; STS = superior temporal sulcus. 


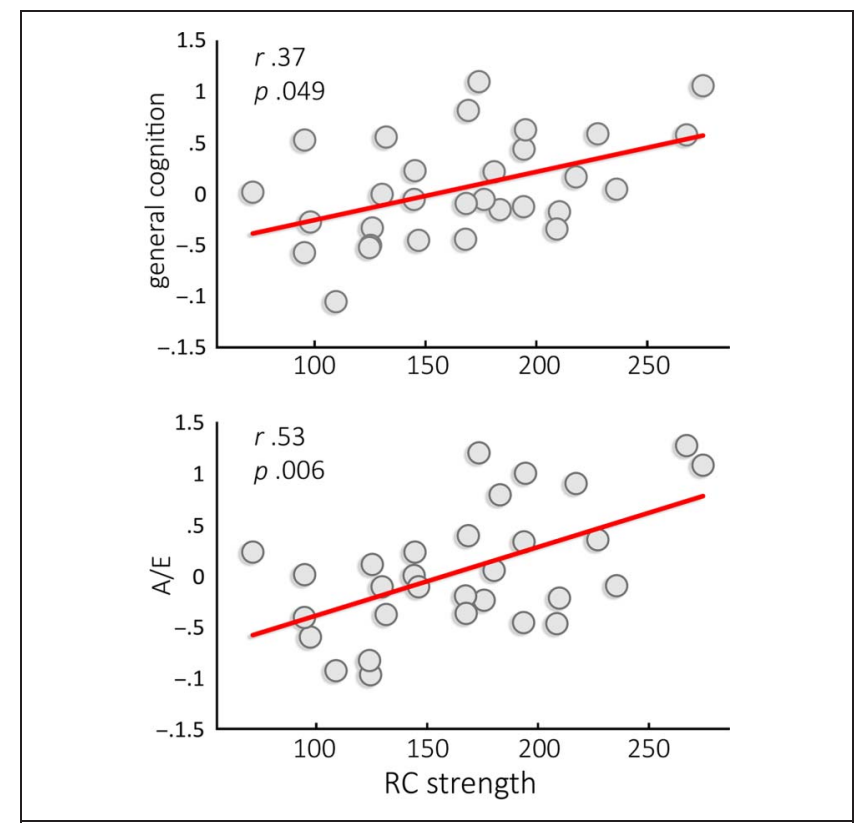

Figure 2. Relationship between rich club strength and performance in general cognitive (top) and $\mathrm{A} / \mathrm{E}$ functions (bottom).

strength and VS/VP scores $(p=.898)$. Additionally, rich club strength correlation with VS/VP scores was significantly weaker than with general cognition and $\mathrm{A} / \mathrm{E}$ scores (respectively, $p=.026$ and $p=.002$, surviving FDR correction). No significant correlations were found between feeder or local edge strength and cognition.

\section{Node and Edge Strength}

As shown in Figure 3A, correlations between general cognition scores and node strength were significantly higher for rich club nodes than non-rich club nodes. A similar pattern was observed with A/E scores. Correlations between edge strength and both general cognition and $\mathrm{A} / \mathrm{E}$ performance were significantly higher in rich club and feeder connections than in local connections (Figure 3B). No significant effects were found for VS/VP scores.

\section{Visual Network versus Rich Club Connectivity Analysis}

Figure $4 \mathrm{~A}$ shows the regions included in the visual network. Correlations with VS/VP scores were significantly stronger for edges between visual network nodes than for edges spanning between non-visual network nodes (Figure 4B). The opposite effect was observed for $\mathrm{A} / \mathrm{E}$ scores, whereas for general cognition scores no significant differences were found (Figure 4B). Additionally, correlations between cognitive scores and node strength were not significantly different between nodes inside and outside the visual network (all $p s>.05$ ).
When comparing the relationship between node and edge strength in the rich club versus those in the visual network, the correlation between connectivity strength and general cognition scores was significantly higher in rich club nodes as compared with nodes in the visual network; a similar effect was observed for A/E scores (Figure 5A). No significant differences were found for VS/VP scores.

The analysis of different edge classes also showed differential effects for all cognitive scores. For A/E and general cognition scores, correlations with rich club edges were significantly stronger than with edges between visual network nodes (Figure 5B). For A/E scores, correlations with feeder edges were also significantly stronger than with edges between visual network nodes (see Figure 5B); a possible similar effect seen for general cognition scores did not survive FDR correction $(p=.026$; FDR-corrected $p=.059$ ). The analysis of VS/VP scores revealed opposite effects. For this cognitive domain, correlations were significantly higher with edges between visual network nodes than with feeder edges (Figure 5B). A potential similar effect between visual network edges and rich club edges did not reach the significance threshold after FDR correction $(p=.025$; FDR-corrected $p=$ $.059)$.

No network connectivity metrics correlated significantly with years of formal education. Also, comparisons of connectivity parameters between genders showed no significant mean differences.

\section{DISCUSSION}

Our study is supportive of the notion of a relationship between human cortical rich club organization and interindividual differences in cognitive performance in healthy older participants. Our findings show structural connectivity strength of nodes and edges inside and outside the rich club to be differentially associated with measures of cognitive performance, being associated with general cognitive and $\mathrm{A} / \mathrm{E}$ performance, but not with performance in the VS/VP domain.

Extending previous observations on rich club formation (Grayson et al., 2014; Van den Heuvel et al., 2014; Crossley et al., 2013; Van den Heuvel \& Sporns, 2011), our analyses now reveal stronger rich club organization to be associated with better cognitive performance. Post hoc analysis of connection classes showed that, in contrast to noncentral local edges spanning between peripheral nodes, rich club edges (i.e., spanning between rich club nodes) and feeder edges (those connecting rich club nodes to the rest of the network) were the most strongly associated with general cognitive and $\mathrm{A} / \mathrm{E}$ performance. Previous studies have suggested that a large proportion of interregional information traffic goes through the brain hubs that constitute the rich club, forming shortcuts potentially allowing more efficient global network communication 
(De Reus \& van den Heuvel, 2014; Goñi et al., 2014; Mišić et al., 2014; Harriger, van den Heuvel, \& Sporns, 2012). The wide repertoire of human cognitive functions relies on such integration (Bressler, 1995), and our findings suggest that the role therein played by the rich club is relevant for cognitive processing.

As different cognitive functions vary regarding the degree to which their neural correlates are anatomically more localized or more distributed, their dependence on global integration may vary accordingly. $\mathrm{A} / \mathrm{E}$ processes rely on the coordinated activity of anatomically distant structures, constituting a set of intrinsic connectivity networks (e.g., the frontoparietal, dorsal attentional, and default mode networks), the dynamical interplay of which is related to attentional performance in healthy participants (Spreng, Sepulcre, Turner, Stevens, \& Schacter, 2013; Sala-Llonch et al., 2012). Rich club and feeder connections, seen in this study to be associated with A/E performance, have been hypothesized to play a central role in the communication between subcomponents

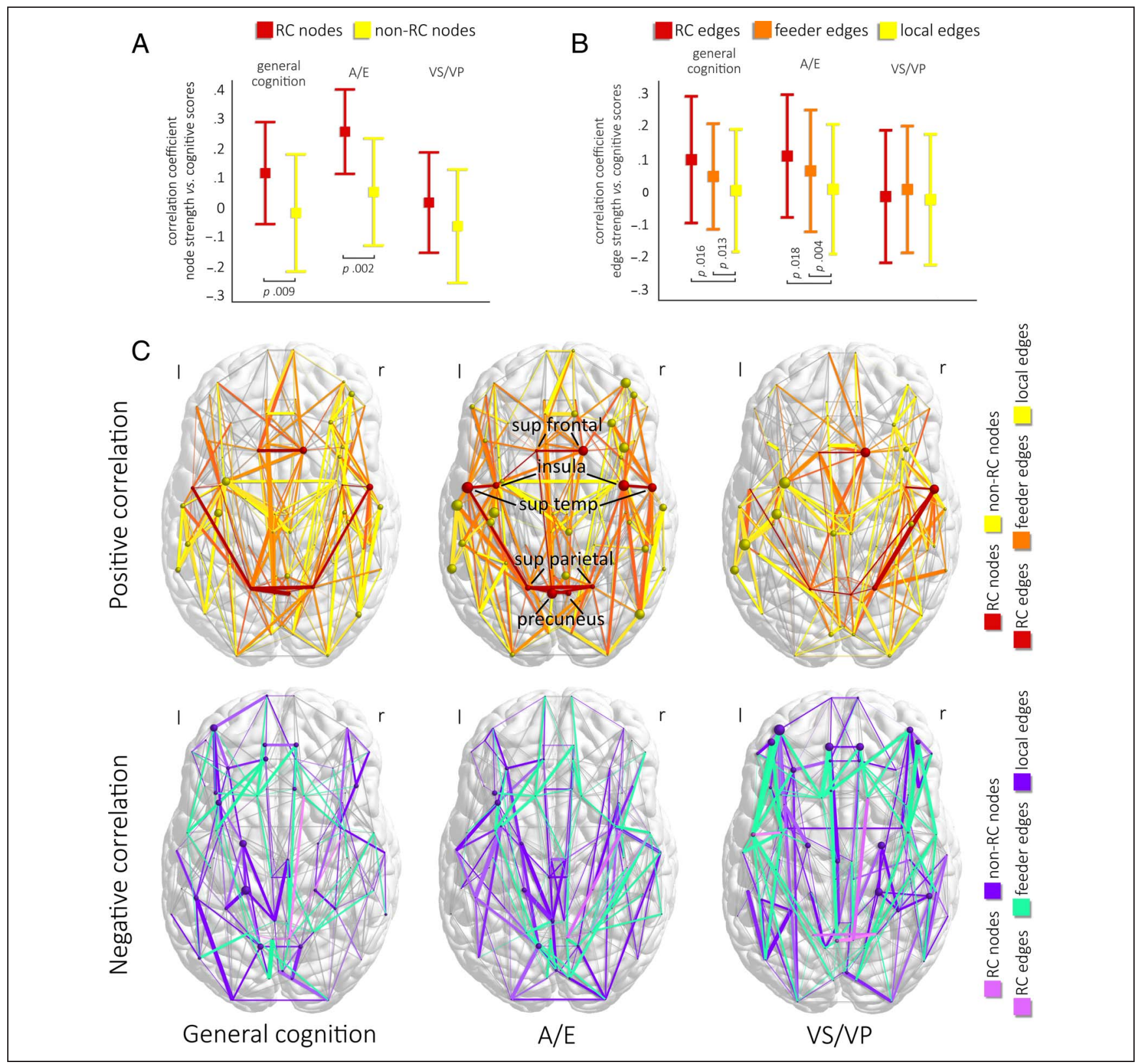

Figure 3. Relationship between rich club (RC) parameters and cognitive performance. (A, B) Plots show the means and standard deviations of coefficients of correlation between cognitive performance and node strength across rich club and non-rich club nodes (A) and between cognitive performance and rich club, feeder, and local edge strength (B). $p$ values for significant (FDR-corrected $p \leq .05$ ) differences are shown. (C) Positive (top row) and negative (bottom row) correlations between edge/node strength and cognitive performance are represented according to class (rich club, feeder, and local); edge thickness and node size are proportional to correlations with general cognition (left), A/E (center), and VS/VP (right) scores. 


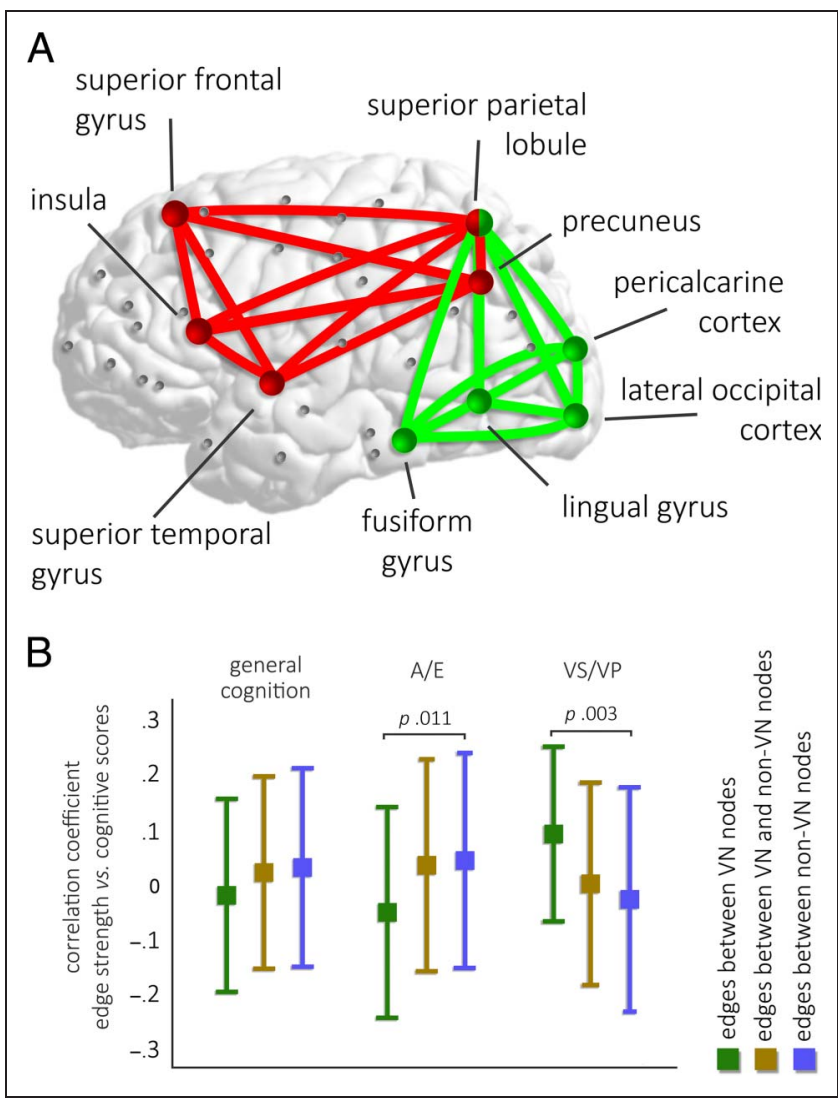

Figure 4. Relationship between visual network (VN) connectivity and cognitive performance. (A) The regions included as nodes of the VN (green) as well as those defined as the rich club (red). The superior parietal lobule was included in both networks. (B) Plots show the means and standard deviations of coefficients of correlation between cognitive performance and strength across edges spanning between VN nodes, edges between VN and non-VN nodes, and edges linking non-VN nodes. $p$ values for significant (FDR-corrected $p \leq .05$ ) differences are shown.

of such networks (Van den Heuvel \& Sporns, 2013a). Combined stronger rich club-to-rich club and rich clubto-non-rich club connections may conceivably allow for more efficient neural communication from peripheral modules - where local processing occurs - to hub regions, with the latter argued to enrich the overall functional repository of the brain at the system's level (Senden et al., 2014).

VS/VP processing, on the other hand, mainly relies on the anatomically localized dorsal and ventral cortical streams (Konen \& Kastner, 2008b), in which neural signal progresses through short-range local connections (Young, 1992). Performance in this domain, associated with connectivity strength in visual network nodes and edges, was not associated with rich club organization, supporting the hypothesized strong involvement of rich club organization in global integrative processes (Van den Heuvel \& Sporns, 2013a).

A sample of relatively older participants (ranging from 39 to 79 years of age) was investigated in this study. Supporting previous studies, we found age-related reductions in global connectivity strength, with no accompanying changes in network density (Fischer, Wolf, Scheurich, \& Fellgiebel, 2014; Gong et al., 2009). As global connectivity changes were not associated with cognitive performance, age-related cognitive decline does not appear to be mediated by them in our sample. Importantly, increasing age was not associated with differential reductions in rich club connectivity strength or with changes in the rich club organization of brain networks. Rich club organization, in turn, explained $\mathrm{A} / \mathrm{E}$ and general cognition performance, suggesting that the effects of rich club connectivity on these cognitive functions are at least partially independent from aging processes.

An interesting hypothesis is that some of the cognitive effects of rich club organization in older persons are a reflection of higher cognitive reserve (Stern, 2002). Education and composite reserve scores have been noted to influence WM microstructural parameters in healthy elders (Arenaza-Urquijo et al., 2013; Teipel et al., 2009). Future studies specifically addressing this issue with comprehensive assessments of cognitive reserve proxies may help clarify this point.

Our findings revealed robust effects between rich clubrelated parameters and cognition but no clear effects

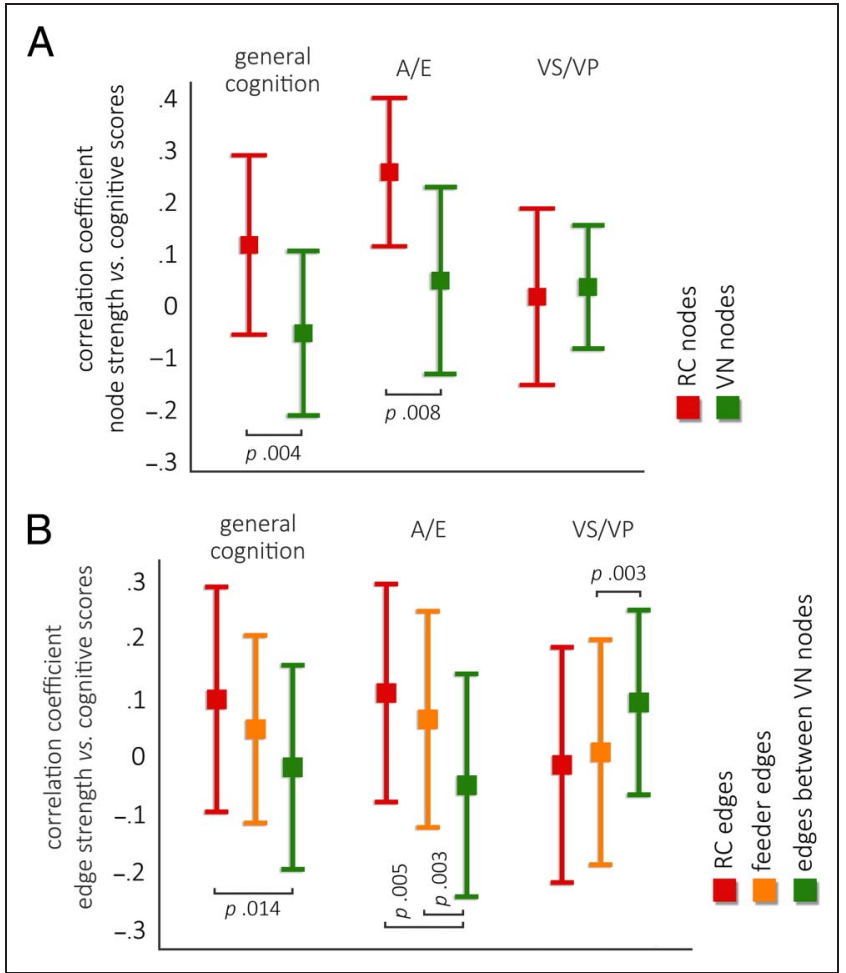

Figure 5. Differential relationship between cognitive performance and connectivity in the rich club (RC) and in the visual network (VN). (A, B) Plots show the means and standard deviations of coefficients of correlation between cognitive performance and node strength across rich club and visual network nodes (A) and between cognitive performance and rich club edges, feeder edges, and edges spanning between visual network nodes (B). $p$ values for significant (FDRcorrected $p \leq .05$ ) differences are shown. 
between global efficiency and cognitive performance are observed (Fischer et al., 2014; Li et al., 2009; Van den Heuvel, Stam, Kahn, \& Hulshoff Pol, 2009). It has been demonstrated that the use of the characteristic path length (a network metric closely related to the global efficiency) may fail to reflect the role of the rich club on brain communication (De Reus \& van den Heuvel, 2014; Irimia \& Van Horn, 2014) and that more refined measures of communication and integration may better characterize the role of the rich club organization in brain networks (De Reus \& van den Heuvel, 2014). Future studies employing these methodologies could help establish whether the relationship between the rich club and cognitive functions is mediated by its role in brain network integration.

Our results seem to indicate that analytic approaches that take into account the rich club organization of brain networks may provide insight into its relationship with normal cognition as well as a potentially useful framework for the study of pathological cognitive decline. In line with these results, studies assessing cognitive impairments of different etiology (e.g., developmental or neurodegenerative; Itahashi et al., 2014; Agosta et al., 2013; Van den Heuvel et al., 2013; Buckner et al., 2009) suggest that hub connectivity plays a role in cognition (Van den Heuvel \& Sporns, 2013b).

Some methodological limitations must be considered. Diffusion MRI offers, at best, an indirect approximation of WM architecture, so its interpretation requires caution. Diffusion imaging-derived measures of connectivity strength can be affected by factors such as fiber orientation coherence, and the biological processes underlying measures such as streamline count are poorly understood (Jones, Knösche, \& Turner, 2013). Nonetheless, most rich club parameters assessed were obtained from intrasubject ratios, thereby potentially reducing the influence of intersubject variation of confound factors on our findings. Also, the use of parcellation schemes with heterogeneous regional sizes (with larger regions potentially having higher degrees) may raise concern about a potential bias in rich club definition. However, it has been shown that regional volume effects do not fully explain rich club formation (Van den Heuvel et al., 2013). The overlap between rich club regions identified through neuroimaging techniques and those described through tract tracing in other mammals further supports this notion (Scholtens, Schmidt, de Reus, \& van den Heuvel, 2014; De Reus \& van den Heuvel, 2013b; Harriger et al., 2012). Importantly, the rich club organization described in our study is consistent with these and other (Van den Heuvel, Scholtens, \& de Reus, 2015; Towlson et al., 2013; Zamora-López, Zhou, \& Kurths, 2009) animal tracing studies. A number of these tract tracing and MRI studies also report on rich club edges displaying high levels of connection strength (Van den Heuvel et al., 2015; Collin et al., 2014), supporting the overall biological validity of rich club formation in the mammalian cortex.
In this study, we have found evidence to support the hypothesis that the rich club plays a relevant role in cognitive processing. Variation in rich club structural connectivity patterns may be related to interindividual differences in global cognitive performance as well as in A/E skills.

\section{Acknowledgments}

This work was funded by the Spanish Ministry of Science and Innovation (PSI2013-41393 grant to C. J., H. C. B., and B. S.), the Generalitat de Catalunya (2014SG98), an FI-DGR grant (2011FI_B 00045 to H. C. B.), and CIBERNED. Martijn van den Heuvel was supported by a VENI (\#451-12-001) grant of the Netherlands Organization for Scientific Research (NWO).

Reprint requests should be sent to Dr. Hugo C. Baggio, Department of Psychiatry and Clinical Psychobiology, University of Barcelona, Casanova 143 (08036) Barcelona, Spain, or via e-mail: hbaggio@ ub.edu.

\section{REFERENCES}

Agosta, F., Sala, S., Valsasina, P., Meani, A., Canu, E., Magnani, G., et al. (2013). Brain network connectivity assessed using graph theory in frontotemporal dementia. Neurology, 81, 134-143.

Arenaza-Urquijo, E. M., Landeau, B., La Joie, R., Mevel, K., Mézenge, F., Perrotin, A., et al. (2013). Relationships between years of education and gray matter volume, metabolism and functional connectivity in healthy elders. Neuroimage, 83, 450-457.

Benjamini, Y. (2010). Discovering the false discovery rate. Journal of the Royal Statistical Society: Series B, Statistical Methodology, 72, 405-416.

Bressler, S. L. (1995). Large-scale cortical networks and cognition. Brain Research. Brain Research Reviews, 20, 288-304.

Bressler, S. L., \& Menon, V. (2010). Large-scale brain networks in cognition: Emerging methods and principles. Trends in Cognitive Sciences, 14, 277-290.

Buckner, R. L., Sepulcre, J., Talukdar, T., Krienen, F. M., Liu, H., Hedden, T., et al. (2009). Cortical hubs revealed by intrinsic functional connectivity: Mapping, assessment of stability, and relation to Alzheimer's disease. The Journal of Neuroscience, 29, 1860-1873.

Bullmore, E., \& Sporns, O. (2009). Complex brain networks: Graph theoretical analysis of structural and functional systems. Nature Reviews Neuroscience, 10, 186-198.

Cao, Q., Shu, N., An, L., Wang, P., Sun, L., Xia, M.-R., et al. (2013). Probabilistic diffusion tractography and graph theory analysis reveal abnormal white matter structural connectivity networks in drug-naive boys with attention deficit/hyperactivity disorder. The Journal of Neuroscience, 33, 10676-10687.

Chang, L., Walker, L., \& Pierpaoli, C. (2012). Informed RESTORE: A method for robust estimation of diffusion tensor from low redundancy datasets in the presence of physiological noise artifacts. Magnetic Resonance in Medicine, 68, 1654-1663.

Collin, G., Kahn, R. S., de Reus, M. A., Cahn, W., \& van den Heuvel, M. P. (2014). Impaired rich club connectivity in unaffected siblings of schizophrenia patients. Schizophrenia Bulletin, 40, 438-448.

Crossley, N. A., Mechelli, A., Vértes, P. E., Winton-Brown, T. T., Patel, A. X., Ginestet, C. E., et al. (2013). Cognitive relevance 
of the community structure of the human brain functional coactivation network. Proceedings of the National Academy of Sciences, U.S.A., 110, 11583-11588.

De Reus, M. A., Saenger, V. M., Kahn, R. S., \& van den Heuvel, M. P. (2014). An edge-centric perspective on the human connectome: Link communities in the brain. Philosophical Transactions of the Royal Society of London, Series B, Biological Sciences, 369. doi:10.1098/rstb.2013.0527.

De Reus, M. A., \& van den Heuvel, M. P. (2013a). Estimating false positives and negatives in brain networks. Neuroimage, 70, 402-409.

De Reus, M. A., \& van den Heuvel, M. P. (2013b). Rich club organization and intermodule communication in the cat connectome. The Journal of Neuroscience, 33, 19929-19939.

De Reus, M. A., \& van den Heuvel, M. P. (2014). Simulated rich club lesioning in brain networks: A scaffold for communication and integration? Frontiers in Human Neuroscience, 8, 647.

Fischer, F. U., Wolf, D., Scheurich, A., \& Fellgiebel, A. (2014). Association of structural global brain network properties with intelligence in normal aging. PloS One, 9, e86258.

Fischl, B., van der Kouwe, A., Destrieux, C., Halgren, E., Ségonne, F., Salat, D. H., et al. (2004). Automatically parcellating the human cerebral cortex. Cerebral Cortex, 14, $11-22$.

Gillebert, C. R., Caspari, N., Wagemans, J., Peeters, R., Dupont, P., \& Vandenberghe, R. (2013). Spatial stimulus configuration and attentional selection: Extrastriate and superior parietal interactions. Cerebral Cortex, 23, 2840-2854.

Gong, G., Rosa-Neto, P., Carbonell, F., Chen, Z. J., He, Y., \& Evans, A. C. (2009). Age- and gender-related differences in the cortical anatomical network. The Journal of Neuroscience, 29, 15684-15693.

Goñi, J., van den Heuvel, M. P., Avena-Koenigsberger, A., Velez de Mendizabal, N., Betzel, R. F., Griffa, A., et al. (2014). Resting-brain functional connectivity predicted by analytic measures of network communication. Proceedings of the National Academy of Sciences, U.S.A., 111, 833-838.

Grayson, D. S., Ray, S., Carpenter, S., Iyer, S., Dias, T. G. C., Stevens, C., et al. (2014). Structural and functional rich club organization of the brain in children and adults. PloS One, 9, e88297.

Hagmann, P., Cammoun, L., Gigandet, X., Meuli, R., Honey, C. J., Wedeen, V. J., et al. (2008). Mapping the structural core of human cerebral cortex. PLoS Biology, 6, e159.

Harriger, L., van den Heuvel, M. P., \& Sporns, O. (2012). Rich club organization of macaque cerebral cortex and its role in network communication. PloS One, 7, e46497.

Irimia, A., \& Van Horn, J. D. (2014). Systematic network lesioning reveals the core white matter scaffold of the human brain. Frontiers in Human Neuroscience, 8, 51.

Itahashi, T., Yamada, T., Watanabe, H., Nakamura, M., Jimbo, D., Shioda, S., et al. (2014). Altered network topologies and hub organization in adults with autism: A resting-state fMRI study. PloS One, 9, e94115.

Jones, D. K., Knösche, T. R., \& Turner, R. (2013). White matter integrity, fiber count, and other fallacies: The do's and don'ts of diffusion MRI. Neuroimage, 73, 239-254.

Konen, C. S., \& Kastner, S. (2008a). Two hierarchically organized neural systems for object information in human visual cortex. Nature Neuroscience, 11, 224-231.

Konen, C. S., \& Kastner, S. (2008b). Two hierarchically organized neural systems for object information in human visual cortex. Nature Neuroscience, 11, 224-231.

Lezak, M. D. (2004). Neuropsychological assessment. Oxford, UK: Oxford University Press.

Li, Y., Liu, Y., Li, J., Qin, W., Li, K., Yu, C., et al. (2009). Brain anatomical network and intelligence. PLoS Computational Biology, 5, e1000395.
McIntosh, A. R. (2000). Towards a network theory of cognition. Neural Networks, 13, 861-870.

Mesulam, M. M. (1998). From sensation to cognition. Brain: A Journal of Neurology, 121, 1013-1052.

Milner, A., \& Goodale, M. (1995). The visual brain in action (1st ed.). Oxford, UK: Oxford University Press.

Mišić, B., Sporns, O., \& McIntosh, A. R. (2014). Communication efficiency and congestion of signal traffic in large-scale brain networks. PLoS Computational Biology, 10, e1003427.

Mori, S., \& van Zijl, P. C. M. (2002). Fiber tracking: Principles and strategies-A technical review. NMR in Biomedicine, 15, 468-480.

Opsahl, T., Colizza, V., Panzarasa, P., \& Ramasco, J. J. (2008). Prominence and control: The weighted rich-club effect. Physical Review Letters, 101, 168702.

Rubinov, M., \& Sporns, O. (2010). Complex network measures of brain connectivity: Uses and interpretations. Neuroimage, 52, 1059-1069.

Sala-Llonch, R., Peña-Gómez, C., Arenaza-Urquijo, E. M., VidalPiñeiro, D., Bargalló, N., Junqué, C., et al. (2012). Brain connectivity during resting state and subsequent working memory task predicts behavioural performance. Cortex, 48 , 1187-1196.

Scholtens, L. H., Schmidt, R., de Reus, M. A., \& van den Heuvel, M. P. (2014). Linking macroscale graph analytical organization to microscale neuroarchitectonics in the macaque connectome. The Journal of Neuroscience, 34 , 12192-12205.

Senden, M., Deco, G., de Reus, M. A., Goebel, R., \& van den Heuvel, M. P. (2014). Rich club organization supports a diverse set of functional network configurations. Neuroimage, 96C, 174-182.

Sporns, O., Honey, C. J., \& Kötter, R. (2007). Identification and classification of hubs in brain networks. PloS One, 2, e1049

Spreng, R. N., Sepulcre, J., Turner, G. R., Stevens, W. D., \& Schacter, D. L. (2013). Intrinsic architecture underlying the relations among the default, dorsal attention, and frontoparietal control networks of the human brain. Journal of Cognitive Neuroscience, 25, 74-86.

Stern, Y. (2002). What is cognitive reserve? Theory and research application of the reserve concept. Journal of the International Neuropsychological Society, 8, 448-460.

Teipel, S. J., Meindl, T., Wagner, M., Kohl, T., Bürger, K., Reiser, M. F., et al. (2009). White matter microstructure in relation to education in aging and Alzheimer's disease. Journal of Alzheimer's Disease, 17, 571-583.

Towlson, E. K., Vértes, P. E., Ahnert, S. E., Schafer, W. R., \& Bullmore, E. T. (2013). The rich club of the C. elegans neuronal connectome. The Journal of Neuroscience, 33, 6380-6387.

Van den Heuvel, M. P., \& Hulshoff Pol, H. E. (2010). Exploring the brain network: A review on resting-state fMRI functional connectivity. European Neuropsychopharmacology, 20, 519-534.

Van den Heuvel, M. P., Kahn, R. S., Goñi, J., \& Sporns, O. (2012). High-cost, high-capacity backbone for global brain communication. Proceedings of the National Academy of Sciences, U.S.A., 109, 11372-11377.

Van den Heuvel, M. P., Kersbergen, K. J., de Reus, M. A., Keunen, K., Kahn, R. S., Groenendaal, F., et al. (2014). The neonatal connectome during preterm brain development. Cerebral Cortex, 1-14. doi:10.1093/cercor/bhu095.

Van den Heuvel, M. P., Scholtens, L. H., \& de Reus, M. A. (2015). Topological organization of connectivity strength in the rat connectome. Brain Structure E Function. doi:10.1007/s00429-015-0999-6. 
Van den Heuvel, M. P., \& Sporns, O. (2011). Rich-club organization of the human connectome. The Journal of Neuroscience, 31, 15775-15786.

Van den Heuvel, M. P., \& Sporns, O. (2013a). An anatomical substrate for integration among functional networks in human cortex. The Journal of Neuroscience, 33, 14489-14500.

Van den Heuvel, M. P., \& Sporns, O. (2013b). Network hubs in the human brain. Trends in Cognitive Sciences, 17, 683-696.

Van den Heuvel, M. P., Sporns, O., Collin, G., Scheewe, T., Mandl, R. C. W., Cahn, W., et al. (2013). Abnormal rich club organization and functional brain dynamics in schizophrenia. JAMA Psychiatry, 70, 783-792.

Van den Heuvel, M. P., Stam, C. J., Kahn, R. S., \& Hulshoff Pol, H. E. (2009). Efficiency of functional brain networks and intellectual performance. The Journal of Neuroscience, 29, 7619-7624

Young, M. P. (1992). Objective analysis of the topological organization of the primate cortical visual system. Nature, $358,152-155$.

Zamora-López, G., Zhou, C., \& Kurths, J. (2009). Graph analysis of cortical networks reveals complex anatomical communication substrate. Chaos (Woodbury, N.Y.), 19, 015117. 